\title{
EVALUATION OF PRODUCTION EFFECTIVENESS IN GARMENT COMPANIES THROUGH KEY PERFORMANCE INDICATORS
}

\author{
S. Spahija, E. Shehi, G. Guxho \\ Textile and Fashion Department, Tirana, Albania \\ sspahija@fim.edu.al, ermira.shehi@upt-tekstilmoda.org, genti.guxho@upt-tekstilmoda.org
}

\begin{abstract}
:
Key Performance Indicators help an organisation to improve productivity and to evaluate its success. Improved productivity is an essential part of increasing the value-added services that companies can offer to clients and hence their competitiveness. By using Key Performance Indicators, a company can establish baseline figures against a number of important areas. They can be considered like a health check on a company or a diagnosis of where a company can improve its performance. The definition of what they are and how they are measured does not change often, but Key Performance Indicators may change as the organisation's goals change. In this paper we present and discuss the way to establish Key Performance Indicators to evaluate the production effectiveness of garment companies. The KPls are being tested in several garment companies in Albania and actually implemented in 25 companies.
\end{abstract}

\section{Key words:}

Key Performance Indicators, effectiveness of production, productivity.

\section{Introduction}

The main goal of this paper is the practical application of a method for evaluating the production effectiveness of garment production companies in the country. The evaluation of production effectivenness is the process by which the company sets the parameters for reaching the required goals. There are 15 indicators against which data should be recorded and that will allow the management of a company to make informed decisions on ways to improve the productivity levels of their organisation.

\section{Methodics and analytical methods used}

To complete the main goal of the paper, data on Key Performance Indicators (KPIs) are collected, and evaluated. After this, suggestions for improvements are presented. The $\mathrm{KPI}$ report acts as a health check on the performance of the company by providing results in the following areas. This, in turn, leads to a diagnosis of what action needs to be taken to achieve improvements. In order to improve productivity there is a need to establish baseline figures against which to monitor improvements.

A Performance Indicator or Key Performance Indicator (KPI) is an industry jargon term for a type of Measure of Performance. KPIs are commonly used by an organisation to evaluate its success or the success of a particular activity in which it is engaged. Sometimes success is defined in terms of making progress towards strategic goals, but often success is simply the repeated achievement of some level of operational goal (zero defects, 10/10 customer satisfaction, etc.). Accordingly, choosing the right KPIs depends upon having a good understanding of what is important to the organisation. The KPIs are an element of the Information Management System of an organisation that need to be calculated and measured on a weekly basis. The KPIs give the results of the company's performance and provide indicators of where their performance should be improved in the future.
Some organisations prefer to register this information on a monthly basis instead of a weekly basis, but this is a long period because for the KPls the action needs to be taken very quickly so that the impact is high. Every company/organisation that collects data applies three rules:

1. Only the registered data are to be collected.

2. No need to register the data if no action is to be undertaken later.

3. No need to analyse the data if no action will be undertaken.

As with all Management Information Systems, the accuracy of the data collected is very important.

\section{Evaluation of production effectivenesss}

- The process through which a company sets the parameters within which all the programs, investments and profits should achieve the desired result.

- Use of statistical data to define the progress path through the specified goals

A Performance Indicator or Key Performance Indicator (KPI) is a term for a type of evaluation of production effectiveness [1]. The KPIs give the results of the performance of a company for the previous week and provide indicators of where action needs to be taken to improve its future performance.

KPIs are commonly used by an organisation to evaluate its success or the success of a particular activity in which it is engaged. Sometimes success is defined in terms of making progress towards strategic goals [2], but often success is simply the repeated achievement of some level of operational goal (zero defects, 10/10 customer satisfaction, etc.). Because of the need to develop a good understanding of what is important, performance indicator selection is often closely associated with the use of various techniques to assess the present state of the business, and its key activities. These assessments often lead to the identification of potential improvements, and as a consequence, performance indicators 
are routinely associated with 'performance improvement' initiatives.

The Key Performance Indicators (KPIs) form an important element of the Management Information System (MIS) of an organisation and should be calculated on a weekly basis. KPIs give results of the company performance of one week before and provide indicators of where to take care to improve the performance.

Some organisations prefer to register this information every month instead of every week, but this is a long period, since action on the KPIs must be taken very quickly in order to achieve more impact. The KPI report acts as a health check on the performance of the company by providing results in the following areas, which in turn leads to a diagnosis of what action needs to be taken to achieve improvements:

Productivity - efficiency of the operators, including utilisation, Company structure - ratio of direct operators to indirect operators,

Human resources - levels of absenteeism and labour turnover Efficient use of resources - fabric and trim utilisation,

Costs - a comparison of the company's Cost per Standard Hour and the Cost per Standard Hour received from the customer,

Quality - Levels of Defects per Hundred Units (DHUs).

There are many reasons why the company should evaluate its production effectiveness:

- To estimate how well it is performing,

- To show how the managers should control the work of the operators,

- To plan the budget: budgets are good means for improving performance,

- To improve the operators' performace,

- To motivate the operators,

- To promote,

- To learn.

\section{Principles of evaluation of production effectiveness}

- The establishment of baseline figures against a number of important areas.

- The measurement of whole work (monitoring the operators).

- If one does not measure the work how one can be informed where there is a need for improvement; how does one know where the operators and money should be allocated?

- Non-measured work should be kept at a minimal level or avoided.

- Monitoring the output each hour/each day.

- Performance evaluation in terms of results is related to the work transformation from managers into an operational one.

- Performance reporting and variations analysis should be regularly registered.

- Regular reporting ensures that correcting actions are undertaken in good time

- Correcting action is important for efficient managerial control

\section{Results}

- The practical and concrete part of the paper through a case study focused on collecting, measuring and analysing the key performance indicators (a total of $13 \mathrm{KPIs}$ ) in one company producing workclothes. The company is located in Tirana, with 10 years of experience in facon production, and is thus exporting finished products to the main destination: German clients. The work is organised in three technological lines. The product is workclothes for some wellknown brand names in Europe.

- The KPIs were collected, measured and analysed for the period April 2011 - December 2011 (40 weeks). The collection and measurement of KPIs was based on a number of useful sheets including Operator Performance sheets, Attendance Records, and Skills Inventory Sheets.

\section{Terminology explanation}

Total Standard Hours produced $=$ Total Standard Minutes earned by the direct operators/ 60

Accurate Standard Minute Values (SMVs) are needed to provide realistic figures. If the SMVs are loose (too high) then the total number of Standard Hours (Std. Hrs.) produced will be inflated and the efficiency will not be a true reflection of the performance. If the SMVs are tight (too low) the reported figures will not be accurate. SMVs are measured against International Standards. This indicator is used for the efficiency of the direct operators.

Total Hours Worked including overtime $=$ Total number of working hours including the extra work hours from direct operators

This is the total number of hours including overtime that all direct operators are at work in the factory. This indicator is used for measuring the efficiency of direct operators.

Number of Direct Operators $=$ Total number of employees involved in the production process where the operations have Standard Minute Values used for the calculation of Total Standard Hours produced

This figure includes: sewing machine operators, pressers, cleaners, cutters, etc.

\section{Efficiency of Direct Operators $=$ Total Number of Standard Hours produced/Total Hours Worked including overtime $X$ $\underline{100 \%}$}

The accuracy of this figure is dependent on the Standard Minutes Values for each operation, accurately measured against International Standards. This is one of the most important KPIs and should be subject to continuous scrutiny by managers to understand what has contributed to the \% reported and how different aspects can be improved. Managers should know daily the level of line/factory efficiency so that immediate action can be taken to drive improvements.

\section{Utilisation of direct operators $=$ (Total Hours Worked including overtime(2) - Off-Standard Hours) / Total Hours Worked including overtime (\%)}

Off-Standard Time is the minutes/hours spent by direct operators when they are not producing Standard Minutes. This $\mathrm{KPI}$ is important in calculating the amount of time that a direct operator spends actually working when he/she is in the factory. In the case of a sewing operator, the Off-Standard Time would include time spent waiting for work, machine breakdown, sewing samples, etc.

\section{Number of Indirect Operators = Total Number of Employees - Number of Direct Operators (3)}

Indirects are all employees not producing Standard Minutes and include everyone from directors to maintenance personnel. 
This number is used for calculation of the ratio of direct and indirect operators. From the International Standards, this ratio should not exceed 1:0.5.

Absenteeism $=$ Total number of hours absent from work by direct operators during the week/ (Total Number of Direct Operators x Number of working hours in the week) (\%)

According to the standards, this figure should not exceed $5 \%$. It is management's responsibility to check the absenteeism level.

Labour Turnover $=$ Total number of employees that have left the company during the week/ Total number of employees at the beginning of the week (\%)

In this calculation the new employees that have joined the company during the week should not be included. According to the standards, this value should not exceed $2 \%$.

\section{Number of styles produced in a week}

The number of style changes experienced during a week can have an impact on the Total Standard Hours produced (1) and the Efficiency of Direct Operators (4).

Average Order Size $=$ Total number of units in each order being produced during the week/ Number of Styles being produced

The trend is for an increasing number of orders with smaller quantities and, like the Number of Styles (11), it can affect the Total Standard Hours produced (1) and the Efficiency of Direct Operators (4).

Standard hours per style $=$ Total effective hours produced in a week/Number of styles produced

The number of standard hours per style for a garment indicates the complexity of the style.

Cost per standard hours = Total Company costs for the week including overheads/ Total Standard Hours produced (1)

This figure includes all factory costs (overheads) to determine the actual cost of producing one Standard Hour of work.

Price $=$ Price from the client for one piece of garment/value of effective minutes for one piece of garment X 60

Average Defects per Hundred Units (DHUs) "In Line" = Number of garments with faults needing repair/ Number of garments examined (\%)

"In Line" refers to a control point that is set up to monitor the quality of the product at a stage during the production.

Average Defects per Hundred Units (DHUs) "End of Line" = Number of garments with faults needing repair/ Number of garments examined (\%)

"End of Line" refers to a control point that is set up to monitor the quality of the garment after manufacturing is complete.

\section{Analysis of the collected data}

The data was collected for a 40 week period at the "Manifaktura 7 " company in Tirana. The company has 10 years of experience in the garment production sector for German clients. They produce workclothes for wellknown brand names in Germany. The analysis of the weekly KPIs report showed that there is a need for qualification in many operations. This may be related to the tendency of the sector to work with small orders and many styles.

Analysis of the KPIs results indicates the following problems and improvements needed:

- An increase of total standard hours produced,

- An increase of about $10 \%$ of efficiency of direct operators (KPI 4),

- An improved level of absenteeism,

- A high number of styles and small orders reflecting the tendency of the garment sector and bringing the need for a

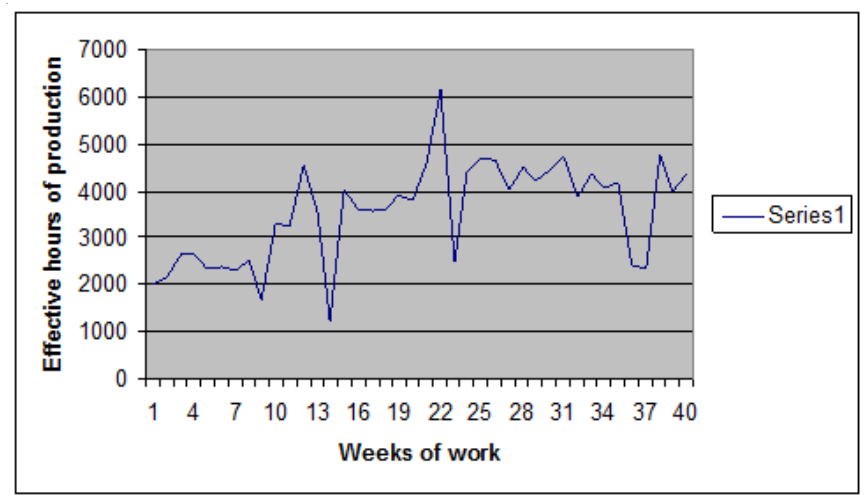

Figure 1. Total standard hours produced.

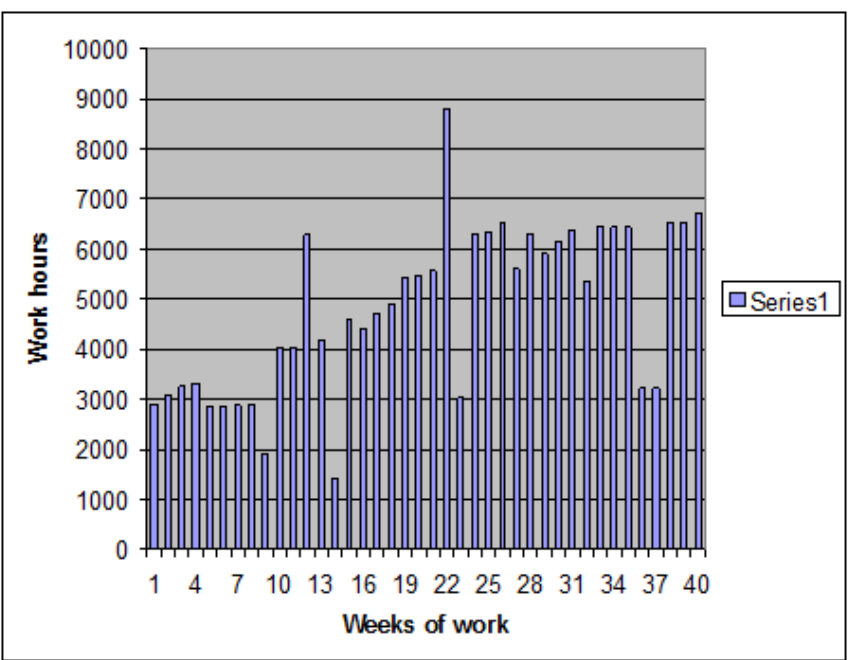

Figure 2. Total hours worked including overtime

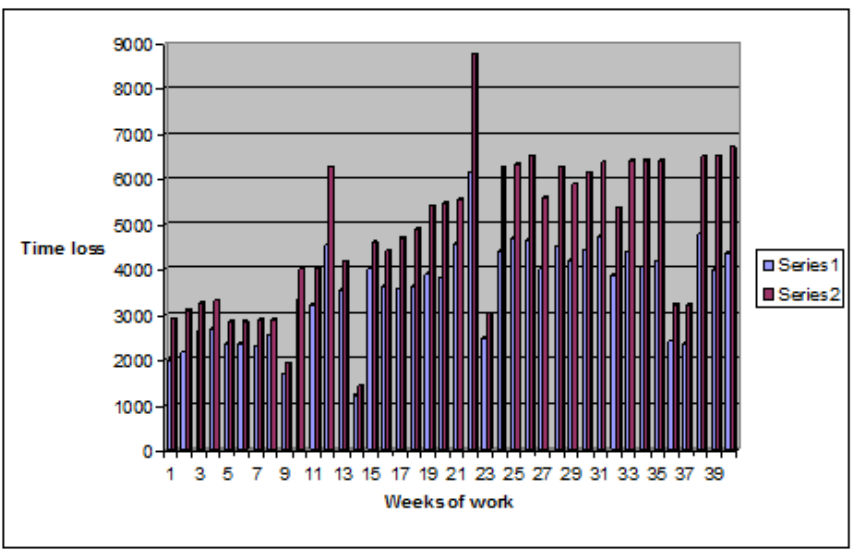

Figure 3. Time loss 
skilled and flexible workforce to maintain good levels of productivity,

- Production of garments with many operations,

- No defects control in line,

- A high percentage of defects at the end of the line were caused by equipment faults and a system needed to be established to identify individual problem machines. The time required for defects repair, thus the extra cost for the company, was high. This was calculated and presented to the management. After this, the tendency was towards improvement of the production quality, reduction of defects at the end of the line and savings.

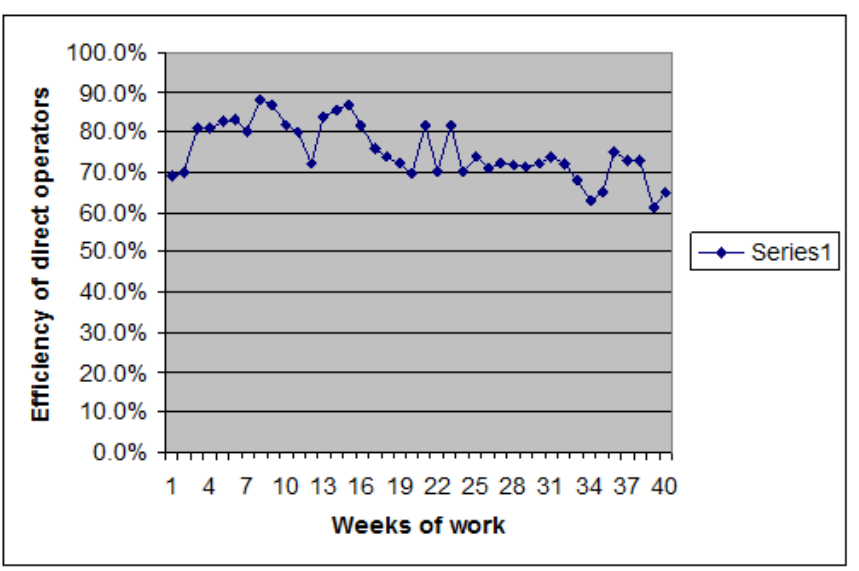

Figure 4. Efficiency of direct operators.

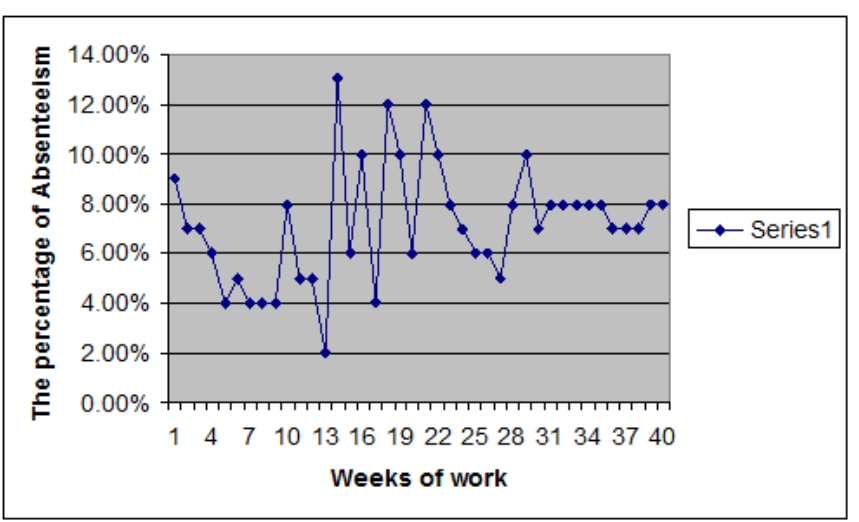

Figure 5. Absenteeism level.

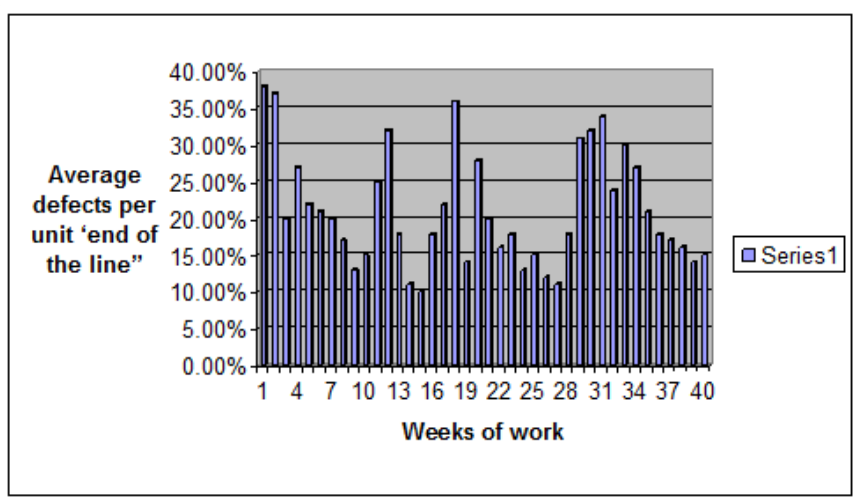

Figure 6. Percentage of defects at the end of the line.

Suggestions for better performance by this company are as follows:

- Analysis of the KPI Reports,

- Review of the accuracy of the data in the KPI Reports,
- Operator training programme,

- Improving individual operator performance,

- Review of the targets being used against International

Standards,

- Improving quality control procedures and reducing defects,

- Delivery of training where appropriate,

- Increased use of IT,

- Training needs analysis should be carried out for all employees,

- Review planning procedures,

- Set up a system to monitor machine breakdown.

\section{Conclusions}

The KPIs reports are like a health check for the company that helps to diagnose the actions to be undertaken in order that improvements take place where needed. For this study 15 indicators for the evaluation of production effectiveness in the garment companies are explained, recommended and calculated to analyse and improve the productivity of the company. The effective minutes are required to get the real figures. If the effective minutes are too high, then the total effective hours produced will be too high as well, and the efficiency will not reflect the performance level. If the effective minutes value is too low the reported figures will not be correct. The value of effective minutes should always be measured according to International Standards. Other performance indicators adopted and used for the productivity and performance evaluation in the garment companies are total number of working hours including extra hours of work; number of indirect and direct operators, the efficiency of direct operators, absenteeism, number of models, average quantity of order, level of defects in line and at the end of the line, average effective hours per model, cost per effective hours, price.

In garment production the time needed for completing different processes is measured in Standard Minutes and should be calculated according to International Standards in order to have accurate data for company performance. Not every company is able to collect and evaluate all the KPIs, but efforts should be made to calculate as many as possible to have maximum benefit.

Managers should know daily the level of company efficiency so that immediate action can be taken to drive improvements. The daily collection of data allows and identifies where the quality problems occur and this should be followed up by Quality Control Personnel with a view to eliminating their cause. The number of total work hours should be under continuous obvservation and control to understand how they contribute to the reported percentages.

Companies need to learn about the problems they have on a weekly basis in order to have the possibility to improve management and productivity, aiming for the desired production cost and profit.

The impact of the number of style changes can be reduced by good planning and multi-skilled operators. About $80 \%$ of the low efficiency is management responsibility. The whole standard time of the break, except for the time spent by machine operators to correct defects, is the responsibility of the management and requires a registration mechanism to collect proper and accurate data.

The currrent tendency is an increase in the order styles and reduction of the quantity per style and this can contribute to the 
total produced effective hours and to the efficiency of direct operators.

The actual cost of the company should be taken into consideration when negotiating the price with clients. Practically, the effective costs should exceed the company cost per effective hour

The 'in line' defects should be monitored carefully in order to know the location of defects and to undertake the proper correcting actions.

Efficiency had risen due to some short training courses undertaken by the skilled operators (more than one operation) and due to the introduction of the policy of 'in line' defects control.

End of line defects remain too high for this type of product. The company needs more qualified operators.

\section{References:}

1. Haycock, John. Productivity toolkit for garment companies. RRije/Albania, USAID, 2011.

2. Hunter, Alan. Quick response in apparel manufacturing.

3. Suanmalu, Suthathip. A study of business performance through Key Performance Indicators (KPIs) in the Thai garment industry.

4. Performance measurement of companies www.wikipedia.org. 